Dataset Paper

\title{
Multiple Ion Cluster Source for the Generation of Magnetic Nanoparticles: Investigation of the Efficiency as a Function of the Working Parameters for the Case of Cobalt
}

\author{
Daniel Llamosa Perez, Lidia Martínez, and Yves Huttel \\ Instituto de Ciencia de Materiales de Madrid, Consejo Superior de Investigaciones Científicas, C/Sor Juana Inés de la Cruz 3, \\ 28049 Madrid, Spain
}

Correspondence should be addressed to Yves Huttel; huttel@icmm.csic.es

Received 26 August 2013; Accepted 27 November 2013; Published 29 April 2014

Academic Editors: S. J. Chua and J. Xu

Copyright (C) 2014 Daniel Llamosa Perez et al. This is an open access article distributed under the Creative Commons Attribution License, which permits unrestricted use, distribution, and reproduction in any medium, provided the original work is properly cited.

\begin{abstract}
We present dataset of Co nanoparticles production using a Multiple Ion Cluster Source (MICS). We study the evolution of the mean size and deposition rate of Co nanoparticles as a function of the power and argon flux applied to the Co magnetron, the aggregation length of the Co magnetron and the total argon flux. The results show the strong influence of these parameters on the mean size of the nanoparticles and the efficiency of the process as well as on the atomic deposition rate. In particular, it is shown that nanoparticles of mean size ranging from 4 to $14 \mathrm{~nm}$ can be produced and that the influence of the working parameters on the production of magnetic nanoparticles is more complex than for the case of noble metal presented previously.
\end{abstract}

\section{Introduction}

One of the ultimate goals in nanotechnology is to provide new systems of production; however, it is reasonable to consider that the success of nanotechnology will rely on its capability of complementing actual technologies before substituting them [1]. The fabrication of nanoscale materials with the expected new properties arising from their reduced size is a prerequisite for their successful use in next generation nanotechnological applications such as magnetic recording, sensing, and biological diagnosis, for example, [2-5]. For these reasons, a great variety of nanoparticle synthesis methods have been developed [6-11].

Among different methods for producing nanoparticles (NPs), the gas-phase synthesis comprises well-known techniques for the production of an extensive variety of nanosized particles [12-15]. These fabrication methods allow the continuous production of clusters with a wide range of sizes (few $\mathrm{nm}$ to tens of $\mathrm{nm}$ ) [16-19]. The gas-phase synthesis processes have been extensively studied [20-24] with a special focus on the nanoparticle yield issues and have become a popular technique for large-scale production and for fundamental studies. On the other hand, gas-phase techniques have the intrinsic added value of producing particles without impurities. As they are generated in vacuum conditions, they are more pure than liquid-based processes since the presence of contaminants from the solution, detrimental for electric and magnetic properties [25-27], is avoided. The presence of impurities can be strongly reduced or even avoided in vacuum and gas-phase systems, which make these techniques the best choice when the NPs purity is critical for specific applications. Haberland et al. [28] developed a gas-phase nanocluster fabrication system using a magnetron sputtering gun to generate the material vapor that is subsequently condensed into nanoparticles and achieved relatively high particle yields. Such model of gas aggregation source or Ion Cluster Source (ICS) has attracted much attention in the last decades for the synthesis of nanoparticles [29]. Argon gas is commonly used as sputtering gas. As soon as a supersaturation condition is reached, the vaporized target atoms coalesce and form the NPs $[19,30-32]$ in the so-called aggregation zone. The nanometer-sized particles exhibit properties that are not found in the corresponding macroscopic systems. Much effort has been recently devoted 
to investigate their size-dependent properties, which offer "tunability" for creating new materials [33-36].

Apart form its vacuum compatibility that guarantees the high purity of the generated NPs, other characteristics of the ICS are the ability (1) to control the size distribution of the fabricated NPs (using a mass filter and/or by controlling the different growth parameters), (2) to be compatible with RF sputtering for the fabrication of NPs from insulating or semiconductor sputtering targets, (3) to allow the injection of other gases such as oxygen and nitrogen for the modification of the composition of the NPs, (4) to control the NPs density by simply adjusting the deposition time, and (5) to allow the deposition of the NPs over any substrate (provided that it is vacuum compatible). While the ICS is very versatile for the fabrication of NPs with the chemical composition identical or very close to that of the sputtering target, it does not allow the fine tune of the chemical composition unless the sputtering target is replaced for each desired stoichiometry. Since the ICS is a vacuum or UHV system, the replacement of the sputtering target can only be done by breaking the vacuum with the subsequent air contamination of the system that needs to be baked (lost of time), and so forth. In order to overcome such limitation, a new kind of gas aggregation source derived from the ICS has been recently proposed [37]. In the new gas aggregation source called Multiple Ion Cluster Source (MICS), the single two-inch magnetron is replaced by 3 one-inch magnetrons that are loaded with different sputtering targets. Since each magnetron can be operated individually (each magnetron has its sputtering gas entry, electrical connection, positioning system, and cooling pipes), the density of ions extracted from each sputtering target can be adjusted and hence the stoichiometry of the resulting NPs [38]. The MICS is now commercially available at Oxford Applied Research Ltd. [39]. However it has been recently reported that, to some extent, the working parameters of the individual magnetrons are correlated through the total pressure inside the aggregation zone and the relative position of the magnetrons [40]. Such correlation has been demonstrated using a single magnetron loaded with a noble metal (silver), where a linear correlation of the NP size and the efficiency with most working parameters was observed. Here we investigate the correlation between the working parameters with a magnetron operated with a magnetic target (cobalt) that is expected to display a different sputtering behavior due to its magnetic properties. We present the evolution of the NP size as well as the efficiency of the process in terms of NP rate and atomic rate, as a function of applied power, argon flux applied to the magnetron, total argon flux, and aggregation length. We observed that the performance of magnetic targets differs from nonmagnetic ones. The efficiency of producing NPs rarely presents a linear tendency as occurred with Ag. Thus, a full description of the evolution as a function of each parameter is needed due to the greater complexity of the system. We will also show that it is possible to generate Co NPs from $4 \mathrm{~nm}$ up to $14 \mathrm{~nm}$ diameter, that is, below and above the superparamagnetic limit that is close to $9 \mathrm{~nm}$ [41] using standard fabrication conditions.

\section{Methodology}

The MICS system is a modified ICS that combines three independent magnetrons into the aggregation zone NC 200U-B model from Oxford Applied Research Ltd. The fabrication of the magnetrons follows the original design of Professor Colino García from the Facultad de Ciencias del Medio Ambiente, Toledo, Spain [42]. Each magnetron possesses its own translation motion, argon mass flow controller, cooling pipes, and electrical connection. Additionally, the main translation that allows a displacement of all the magnetrons into the aggregation zone is preserved like in a standard ICS. More details of the MICS can be found elsewhere [38]. A schematic view of the MICS is shown in Figure 1(a) while Figures 1(b) and 1(c) show pictures of the side and top views of the system. Figure 1(d) displays the configuration of the magnetrons that are placed into the aggregation zone. The MICS was connected to a UHV chamber with a base pressure in the low $10^{-9}$ mbar range.

For the present study, a Co target (99.99\%) of 1 inch diameter was loaded in one of three magnetrons (Figure 1(d)). The other two magnetrons, identified as M2 and M3, were also loaded with target materials but were not used to generate ions in the present study. The magnetron loaded with Co was always positioned closer to the exit diaphragm of the MICS than the other 2 magnetrons in order to avoid target contamination by Co plasma. The NPs generated with the MICS were deposited on flat silicon wafers $\left(10 \times 10 \mathrm{~mm}^{2}\right)$ introduced into UHV through a fast entry load lock and then placed in the chamber at approximately $200 \mathrm{~mm}$ from the exit diaphragm of the MICS. All the deposits were performed at room temperature. Note that the distance between the Si substrate and the magnetron plasma is long enough to avoid any heating effect on the substrate where no change in temperature could be detected. The spot diameter of the area where the NPs landed was $\approx 40 \mathrm{~mm}$. No size distribution was observed in the deposited spot, although some density variations were detected, the center of the deposition spot being covered by a higher density of nanoparticles. In order to follow the evolution of the density of nanoparticles with the working parameters of the MICS, the measurements were always performed in the region close to the center of the deposition spot. The size distributions and deposition rates $\left(\mathrm{NPs} / \mu \mathrm{m}^{2} \mathrm{~s}\right.$ ) were extracted from the analysis of atomic force microscopy (AFM) images. The AFM images were acquired using the Cervantes AFM System equipped with the Dulcinea electronics from Nanotec Electronica SL [43] in dynamic mode using commercial silicon AFM tips with a typical radius less than $7 \mathrm{~nm}$. The WSxM software [44] has been used for the analysis of the images. The size distribution of the NPs was extracted from several AFM images through the NP height measurement. The number of AFM images required for the estimation of the size distribution is dependent on the coverage percentage and ranged from 2 to 11 images. The fit of the height distributions to extract the mean sizes has been performed assuming a Galton or lognormal distribution [45]. The deposition rates were calculated from the count of NPs from several AFM images and the deposition time of each sample. Based on previous studies on similar systems, 
(a)

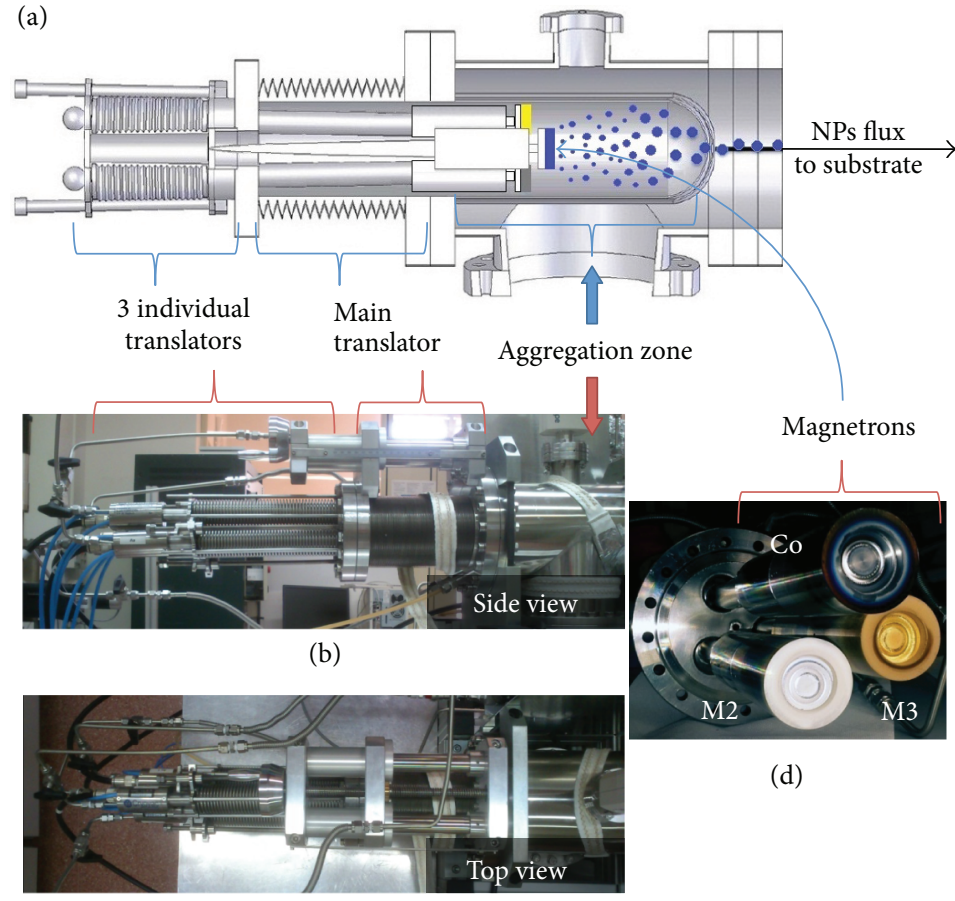

(c)

FIGURE 1: Schematic representation of the MICS (a). Side picture (b) and top picture of the MICS (c). Picture of the magnetrons that are placed in the aggregation zone (d).

the NP height is equivalent to the diameter of NP [46], so it can be assumed that the particles studied in this dataset have spherical shape. Therefore, the atomic deposition rates (atoms $/ \mu \mathrm{m}^{2} \mathrm{~s}$ ) were calculated as the product of the number of atoms per NP (using the volume of a sphere of the NP mean size and the density of cobalt) and the NPs deposition rates.

Different series of NPs deposits have been performed by changing a specific parameter, while keeping the other constant. The parameters that were tuned were the following: $(1)$ the power applied to the Co magnetron $(P),(2)$ the argon flux applied to the Co magnetron $\left(\Phi_{\mathrm{Co}}\right),(3)$ the total argon flux $\left(\Phi_{\text {Total }}\right)$, and (4) the aggregation length (defined as the distance between the magnetron head and the exit diaphragm of the aggregation zone) of the Co magnetron $\left(L_{\mathrm{Co}}\right)$. For all samples the aggregation lengths of magnetrons M2 and M3 were fixed $\left(L_{\mathrm{M} 2}=175 \mathrm{~mm}\right.$ and $\left.L_{\mathrm{M} 3}=172 \mathrm{~mm}\right)$.

For the study of the influence of the power applied to the Co magnetron, two series of samples were produced. For both series $P_{A}$ and $P_{B}$, the same $L_{C o}=150 \mathrm{~mm}$ and $\Phi_{\text {Total }}=80 \mathrm{sccm}$ were used. Moreover, $\Phi_{\mathrm{Co}}=5 \mathrm{sccm}$ and $\Phi_{\text {Co }}=30 \mathrm{sccm}$ were used for series $P_{A}$ and $P_{B}$, respectively (sccm is the standard cubic centimeter per minute at standard temperature and pressure). Also the power applied to the Co magnetron was varied between series of $P_{A}$ and $P_{B}$. In Table 1 , we show the values of the parameters used for both series as well as the obtained results. In Figure 2, we display the evolution of the mean size and deposition rate corresponding to series $P_{A}$ and $P_{B}$. As can be observed, the mean size increases with increasing applied power for both series. On the other hand, the evolution of the deposition rate is not similar for both series. While this rate increases with increasing power for $P_{B}$ (highest $\Phi_{\text {Co }}$ and $P$ ), it does the opposite for series $P_{A}$. Figure 3 displays the evolution of the atomic deposition rate for $P_{A}$ and $P_{B}$. It is shown that the atomic deposition rate can be tuned with both $P$ and $\Phi_{\text {Co }}$. The evolution of the efficiency with the argon flux is further investigated in the following.

For the study of the influence of the argon flux, two series of samples were fabricated and characterized. For series $\Phi_{A}$, $\Phi_{\text {Co }}$ was varied while keeping $\Phi_{\text {Total }}$ constant at $80 \mathrm{sccm}$. For series $\Phi_{B}, \Phi_{\text {Co }}$ was kept fixed at $10 \mathrm{sccm}$ and $\Phi_{\text {Total }}$ was varied. In both cases, $P=5 \mathrm{~W}$ and $L_{\mathrm{C}_{0}}=150 \mathrm{~mm}$. The parameters used for the fabrication and results obtained are given in Table 2 and the corresponding graphs are displayed in Figures 4 and 5. Figure 4(a) clearly shows a decrease of the mean size of the NPs and an increase of the deposition rate with the increasing $\Phi_{\mathrm{Co}}$. Although both evolutions are not linear, the resulting atomic deposition rate displayed in Figure 4(b) presents a linear evolution. This indicates that the number of Co atoms extracted from the MICS steady decreases as a function of $\Phi_{\mathrm{Co}}$. The observed evolution of series $\Phi_{B}$ (Figure 5) is rather different from that of series $\Phi_{A}$. While in both cases we observed an increase of the deposition rate, for series $\Phi_{B}$ the mean size of the NPs also increases with increasing $\Phi_{\text {Total }}$ in opposition to series $\Phi_{A}$. Surprisingly the nonlinear evolutions observed in Figure 5(a) also give rise to a linear evolution of the atomic deposition rate (Figure 5(b)). In opposition to series $\Phi_{A}$ the atomic deposition rate increases with increasing $\Phi_{\text {Total }}$. 
TABLE 1: Fabrication parameters and results as a function of power applied to the Co magnetron.

\begin{tabular}{|c|c|c|c|c|c|c|}
\hline \multicolumn{7}{|c|}{ Series $P_{\mathrm{A}}$} \\
\hline \multirow{2}{*}{ Constant parameters } & \multicolumn{3}{|c|}{$\Phi(\mathrm{sccm})$} & \multicolumn{3}{|c|}{$L(\mathrm{~mm})$} \\
\hline & Co: 5 & M2: 37.5 & M3: 37.5 & Co: 150 & M2: 175 & M3: 172 \\
\hline Power Co $(\mathrm{W})$ & Mean size (nm) & NPs rate $\left(\mathrm{NPs} / \mu \mathrm{m}^{2} \mathrm{~s}\right)$ & At rate $\left(10^{4}\right.$ at $\left./ \mu \mathrm{m}^{2} \mathrm{~s}\right)$ & AFM images and files & \multicolumn{2}{|c|}{ Size distribution file } \\
\hline 4 & $12.4 \pm 1.4$ & $0.23 \pm 0.10$ & $2.1 \pm 0.8$ & Images $1-8$ & \multicolumn{2}{|c|}{ Dataset Item 3} \\
\hline 5 & $13.3 \pm 1.2$ & $0.12 \pm 0.03$ & $1.3 \pm 1.3$ & Images 9-19 & \multicolumn{2}{|c|}{ Dataset Item 4} \\
\hline 6 & $14.8 \pm 0.8$ & $0.06 \pm 0.02$ & $0.9 \pm 0.9$ & Images 20-27 & \multicolumn{2}{|c|}{ Dataset Item 5} \\
\hline \multicolumn{7}{|c|}{ Series $P_{\mathrm{B}}$} \\
\hline \multirow{2}{*}{ Constant parameters } & \multicolumn{3}{|c|}{$\Phi(\mathrm{sccm})$} & \multicolumn{3}{|c|}{$L(\mathrm{~mm})$} \\
\hline & Co: 30 & M2: 25 & M3: 25 & Co: 150 & M2: 175 & M3: 172 \\
\hline Power Co $(\mathrm{W})$ & Mean size $(\mathrm{nm})$ & NPs rate $\left(\mathrm{NPs} / \mu \mathrm{m}^{2} \mathrm{~s}\right)$ & At rate $\left(10^{4}\right.$ at $\left./ \mu \mathrm{m}^{2} \mathrm{~s}\right)$ & AFM images and files & \multicolumn{2}{|c|}{ Size distribution file } \\
\hline 5 & $3.9 \pm 0.3$ & $0.3 \pm 0.1$ & $0.1 \pm 0.2$ & Images 28-29 & \multicolumn{2}{|c|}{ Dataset Item 6} \\
\hline 8 & $5.2 \pm 0.5$ & $0.8 \pm 0.1$ & $0.5 \pm 0.3$ & Images 30-32 & \multicolumn{2}{|c|}{ Dataset Item 7} \\
\hline 10 & $6.0 \pm 0.6$ & $2.5 \pm 0.1$ & $2.5 \pm 0.4$ & Images 33-35 & \multicolumn{2}{|c|}{ Dataset Item 8} \\
\hline 12 & $6.2 \pm 0.8$ & $1.3 \pm 0.2$ & $1.5 \pm 0.7$ & Images 36-39 & \multicolumn{2}{|c|}{ Dataset Item 9} \\
\hline
\end{tabular}

TABLE 2: Fabrication parameters and results as a function of argon flux.

\begin{tabular}{|c|c|c|c|c|c|c|c|}
\hline \multicolumn{8}{|c|}{ Series $\Phi_{\mathrm{A}}$} \\
\hline \multicolumn{3}{|c|}{ Constant parameters } & \multirow{4}{*}{$\begin{array}{c}\text { Power Co }(\mathrm{W}) \\
5 \\
\text { Mean size }(\mathrm{nm})\end{array}$} & \multirow{2}{*}{\multicolumn{4}{|c|}{$L(\mathrm{~mm})$}} \\
\hline & & & & & & & \\
\hline \multicolumn{3}{|c|}{$\Phi(\mathrm{sccm})$} & & \multirow{2}{*}{ NPs rate $\left(\mathrm{NPs} / \mu \mathrm{m}^{2} \mathrm{~s}\right)$} & \multirow{2}{*}{ At rate $\left(10^{4}\right.$ at $\left./ \mu \mathrm{m}^{2} \mathrm{~s}\right)$} & \multirow{2}{*}{ AFM images and files } & \multirow{2}{*}{ Size distribution file } \\
\hline Co & M2 & M3 & & & & & \\
\hline 5 & 37.5 & 37.5 & $13.3 \pm 1.1$ & $0.1 \pm 0.4$ & $1.3 \pm 0.4$ & Images $40-50$ & Dataset Item 10 \\
\hline 20 & 30 & 30 & $6.3 \pm 0.6$ & $1.1 \pm 0.5$ & $1.3 \pm 0.2$ & Images 51-54 & Dataset Item 11 \\
\hline 40 & 20 & 20 & $3.6 \pm 0.8$ & $4.6 \pm 0.8$ & $1.0 \pm 0.1$ & Images 55-57 & Dataset Item 12 \\
\hline \multicolumn{8}{|c|}{ Series $\Phi_{B}$} \\
\hline \multirow{2}{*}{\multicolumn{3}{|c|}{ Constant parameters }} & $\Phi_{\mathrm{Co}}(\mathrm{sccm})$ & Power Co $(\mathrm{W})$ & & $L(\mathrm{~mm})$ & \\
\hline & & & 10 & 5 & M3: 150 & M2: 175 & M3: 172 \\
\hline \multicolumn{3}{|c|}{$\Phi(\mathrm{sccm})$} & Mean size (nm) & NPs rate $\left(\mathrm{NPs} / u \mathrm{~m}^{2} \mathrm{~s}\right)$ & At rate $\left(10^{4} \mathrm{at} / \mu \mathrm{m}^{2} \mathrm{~s}\right)$ & AFM images and files & Size distribution file \\
\hline M2 & & M3 & & & & स⿴囗十 & 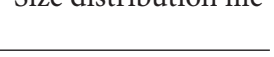 \\
\hline 15 & & 15 & $6.1 \pm 0.7$ & $0.8 \pm 0.2$ & $0.9 \pm 0.6$ & Images 58-63 & Dataset Item 13 \\
\hline 25 & & 25 & $6.6 \pm 1.0$ & $3.0 \pm 0.3$ & $4.1 \pm 1.0$ & Images 64-66 & Dataset Item 14 \\
\hline 35 & & 35 & $7.1 \pm 1.4$ & $4.8 \pm 0.4$ & $8.2 \pm 1.7$ & Images 67-68 & Dataset Item 15 \\
\hline 45 & & 45 & $7.7 \pm 1.0$ & $5.5 \pm 0.9$ & $12.0 \pm 2.8$ & Images $69-71$ & Dataset Item 16 \\
\hline 55 & & 55 & $8.4 \pm 1.0$ & $4.8 \pm 0.8$ & $13.5 \pm 3.3$ & Images 72-74 & Dataset Item 17 \\
\hline
\end{tabular}

The last series of samples has been elaborated in order to study the influence of the aggregation length $L_{\mathrm{Co}}$ of the cobalt. For this series $P=10 \mathrm{~W}, \Phi_{\text {Total }}=80 \mathrm{sccm}$, and $\Phi_{\text {Co }}=30$ sccm were kept constant. The different parameters and results are given in Table 3. From Figure 6(a) it appears that the mean size of the NPs is almost a linear function of the increasing $L_{\mathrm{Co}}$. This behavior is similar to that of a standard ICS although the size range is smaller in the case of the MICS. On the other hand, it is observed that the deposition rate (Figure 6(a)) reaches a maximum and then decreases indicating that the maximum efficiency is obtained at $L_{\mathrm{Co}} \approx 130 \mathrm{~mm}$. The observed behavior of the deposition rate (Figure 6(a)) strongly influences the atomic deposition rate that reaches a maximum at around $L_{\mathrm{Co}} \approx 140 \mathrm{~mm}$ (Figure 6(b)).

\section{Dataset Description}

The dataset associated with this Dataset Paper consists of 22 items which are described as follows.

Dataset Item 1 (Images). Eighty-seven AFM images required for the estimation of the size distribution for nanoparticles deposits. Each deposit has been measured by AFM by 


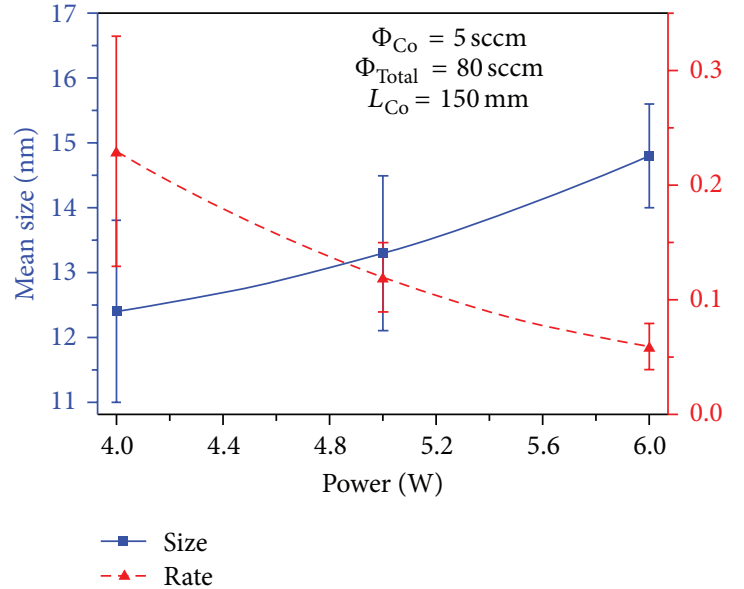

(a)

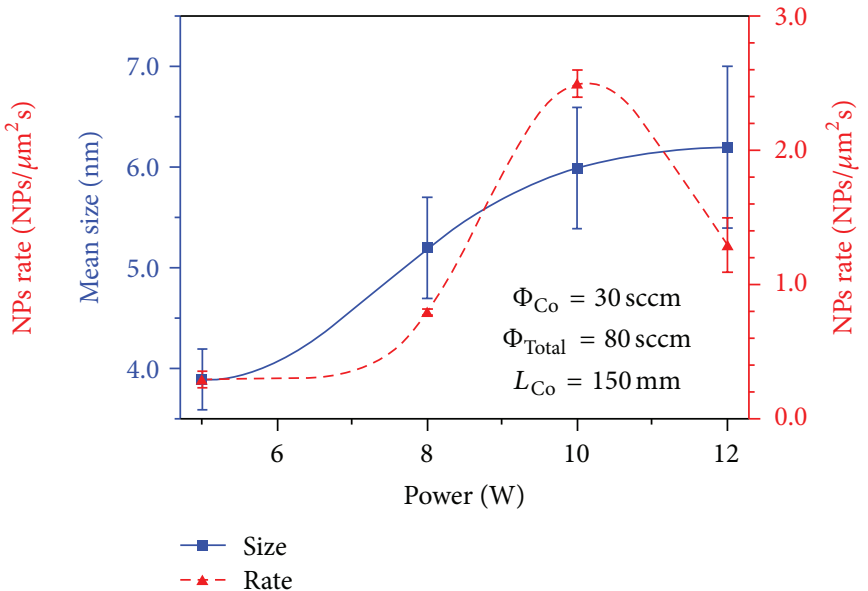

(b)

FIGURE 2: Evolution of the mean size and deposition rate of Co nanoparticles as a function of the power applied to the magnetron and for a fixed argon flux of $5 \mathrm{sccm}$ on the magnetron (a). The same as (a) but with a higher argon flux on the magnetron ( $30 \mathrm{sccm}$ ) and for a higher applied power range (b).

TABLE 3: Fabrication parameters and results as a function of aggregation length of the Co magnetron.

\begin{tabular}{|c|c|c|c|c|c|}
\hline \multirow{2}{*}{ Constant parameters } & \multicolumn{2}{|c|}{$\Phi(\mathrm{sccm})$} & \multirow{2}{*}{$\begin{array}{c}\text { Power Co }(\mathrm{W}) \\
10\end{array}$} & \multicolumn{2}{|c|}{$L(\mathrm{~mm})$} \\
\hline & Co: 30 & M2: 25 & & M2: 175 & M3: 172 \\
\hline$L_{\mathrm{Co}}(\mathrm{mm})$ & Mean size $(\mathrm{nm})$ & NPs rate $\left(\mathrm{NPs} / \mu \mathrm{m}^{2} \mathrm{~s}\right)$ & At rate $\left(10^{4}\right.$ at $\left./ \mu \mathrm{m}^{2} \mathrm{~s}\right)$ & AFM images and files & Size distribution file \\
\hline 170 & $6.4 \pm 0.7$ & $1.7 \pm 0.2$ & $2.1 \pm 0.7$ & Images 75-76 & Dataset Item 18 \\
\hline 150 & $6.0 \pm 0.6$ & $2.5 \pm 0.1$ & $2.6 \pm 0.4$ & Images 77-78 & Dataset Item 19 \\
\hline 120 & $5.7 \pm 0.6$ & $3.3 \pm 0.1$ & $2.9 \pm 0.4$ & Images $79-81$ & Dataset Item 20 \\
\hline 90 & $5.6 \pm 0.4$ & $1.3 \pm 0.1$ & $1.1 \pm 0.3$ & Images $82-84$ & Dataset Item 21 \\
\hline 75 & $5.0 \pm 0.6$ & $0.8 \pm 0.1$ & $0.5 \pm 0.3$ & Images $85-87$ & Dataset Item 22 \\
\hline
\end{tabular}

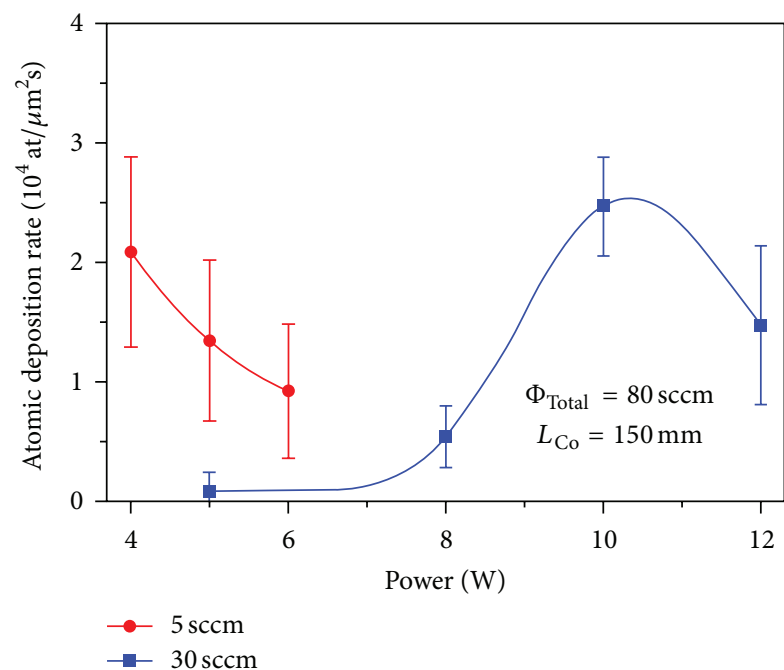

FIGURE 3: Atomic deposition rate as a function of power applied to the magnetron derived from Figure 2.

recording images of scan areas of $1 \times 1 \mu \mathrm{m}^{2}, 2 \times 2 \mu \mathrm{m}^{2}$, and $3 \times 3 \mu \mathrm{m}^{2}$, AFM images were analyzed, and from these images, the height of 4895 nanoparticles was extracted. For each nanoparticles deposit, the fit of the nanoparticles size distribution was performed.

Dataset Item 2 (3D Object Data). Eighty-seven STP files for the full content of Dataset Item 1 (Images) that allow the analysis of the images.

Dataset Item 3 (Table). Size distribution data extracted from images 1-8 in Dataset Item 1.

Column 1: Size (nm)

Column 2: Number of Events

Dataset Item 4 (Table). Size distribution data extracted from images 9-19 in Dataset Item 1.

Column 1: Size (nm)

Column 2: Number of Events

Dataset Item 5 (Table). Size distribution data extracted from images 20-27 in Dataset Item 1.

Column 1: Size (nm)

Column 2: Number of Events 


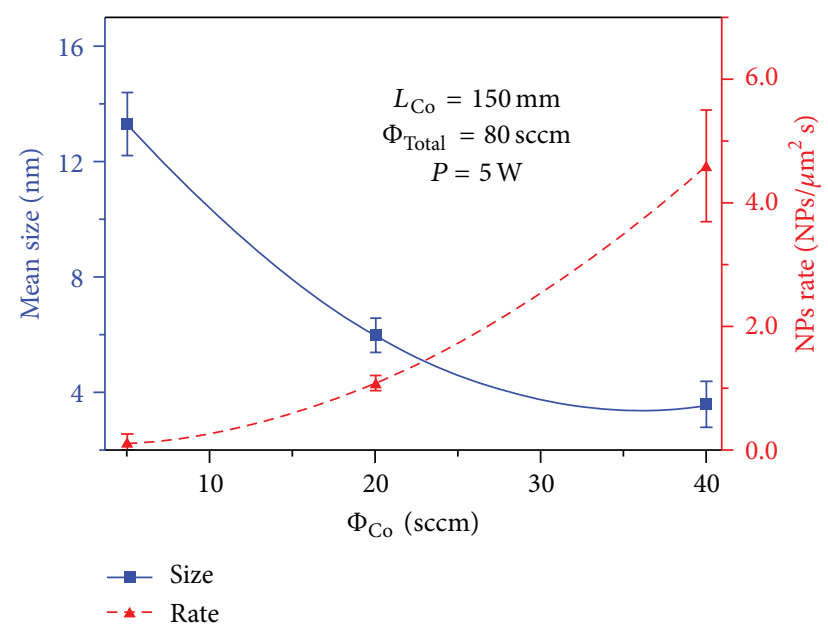

(a)

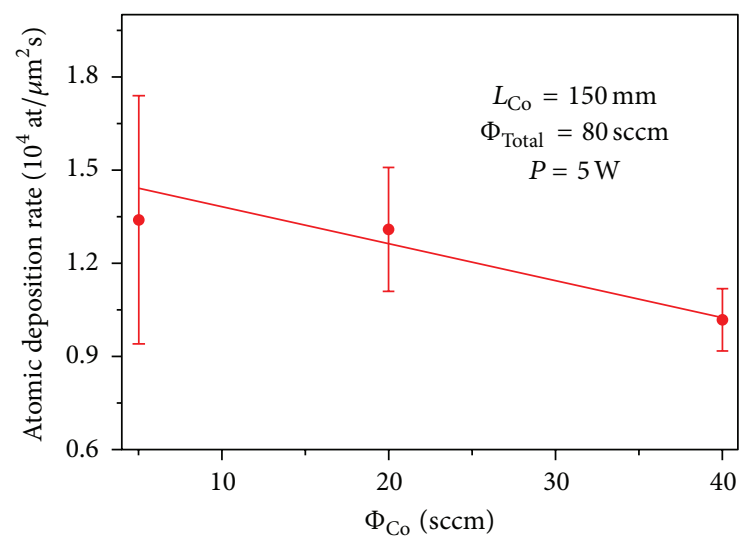

(b)

FIGURE 4: Evolution of the mean size and deposition rate of Co nanoparticles as a function of the argon flux injected into the magnetron and for a fixed total argon flux (a). Atomic deposition rate as a function of the argon flux injected into the magnetron and for a fixed total argon flux (b).

Dataset Item 6 (Table). Size distribution data extracted from images 28-29 in Dataset Item 1.

Column 1: Size (nm)

Column 2: Number of Events

Dataset Item 7 (Table). Size distribution data extracted from images 30-32 in Dataset Item 1.

Column 1: Size (nm)

Column 2: Number of Events

Dataset Item 8 (Table). Size distribution data extracted from images 33-35 in Dataset Item 1.

Column 1: Size (nm)

Column 2: Number of Events
Dataset Item 9 (Table). Size distribution data extracted from images 36-39 in Dataset Item 1.

Column 1: Size (nm)

Column 2: Number of Events

Dataset Item 10 (Table). Size distribution data extracted from images 40-50 in Dataset Item 1.

Column 1: Size (nm)

Column 2: Number of Events

Dataset Item 11 (Table). Size distribution data extracted from images 51-54 in Dataset Item 1.

Column 1: Size (nm)

Column 2: Number of Events

Dataset Item 12 (Table). Size distribution data extracted from images 55-57 in Dataset Item 1.

Column 1: Size (nm)

Column 2: Number of Events

Dataset Item 13 (Table). Size distribution data extracted from images 58-63 in Dataset Item 1.

Column 1: Size (nm)

Column 2: Number of Events

Dataset Item 14 (Table). Size distribution data extracted from images 64-66 in Dataset Item 1.

Column 1: Size (nm)

Column 2: Number of Events

Dataset Item 15 (Table). Size distribution data extracted from images 67-68 in Dataset Item 1.

Column 1: Size (nm)

Column 2: Number of Events

Dataset Item 16 (Table). Size distribution data extracted from images 69-71 in Dataset Item 1.

Column 1: Size (nm)

Column 2: Number of Events

Dataset Item 17 (Table). Size distribution data extracted from images 72-74 in Dataset Item 1.

Column 1: Size (nm)

Column 2: Number of Events 


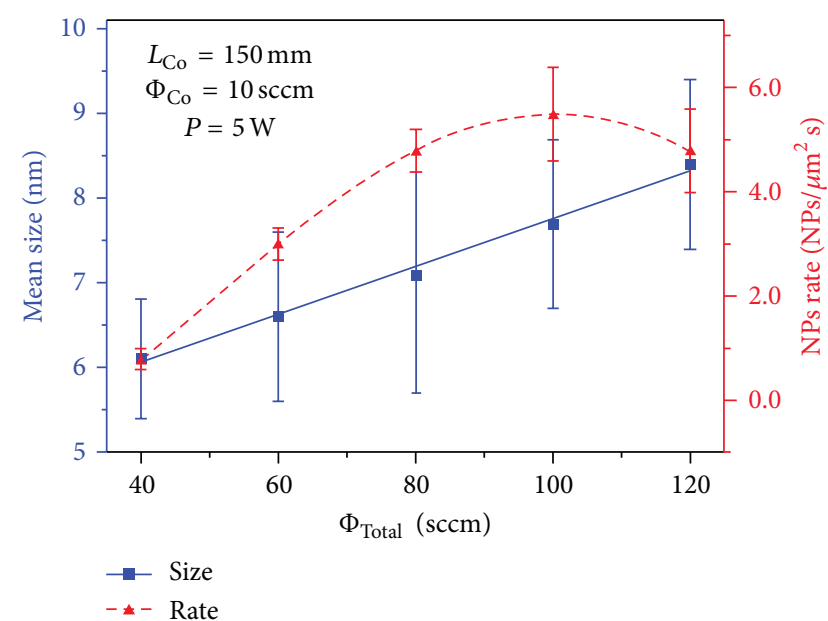

(a)

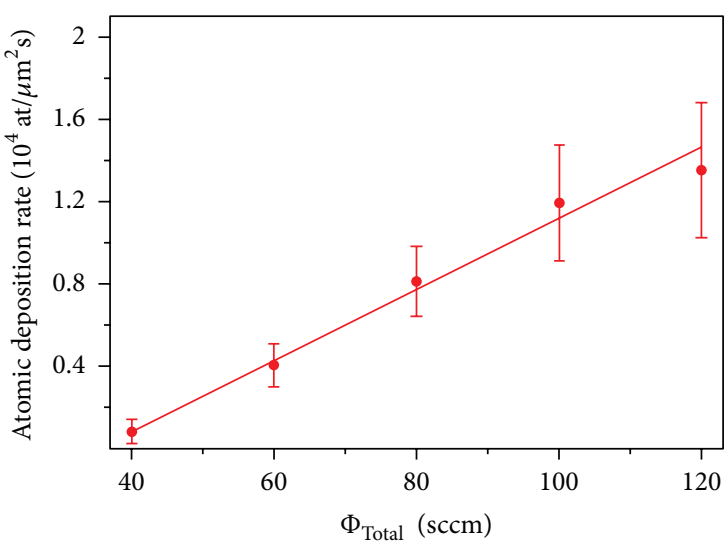

(b)

Figure 5: Evolution of the mean size and deposition rate of Co nanoparticles as a function of the total argon flux while keeping a fixed argon flux into the Co magnetron (a). Atomic deposition rate as a function of the total argon flux while keeping a fixed argon flux into the Co magnetron (b).

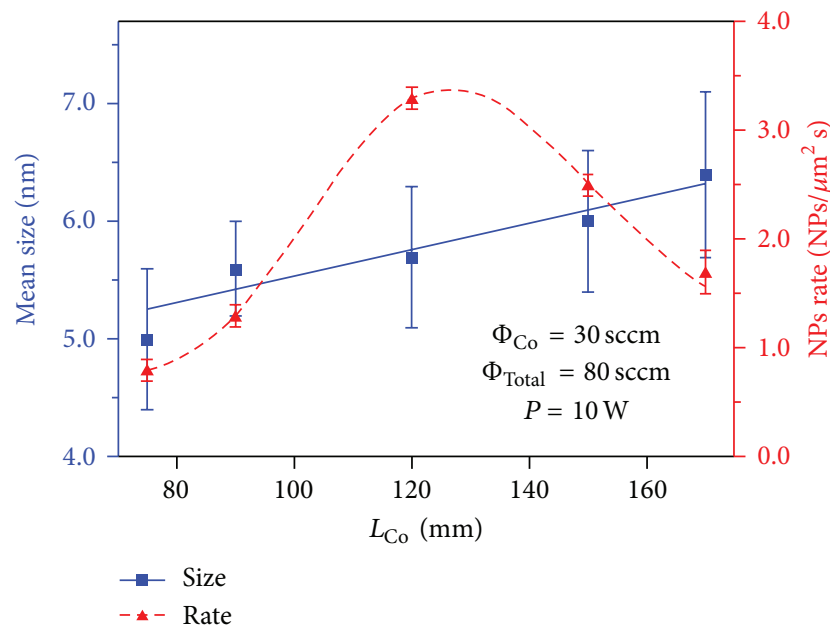

(a)



(b)

FIgURE 6: Evolution of the mean size and deposition rate of Co nanoparticles as a function of the position of the magnetron into the aggregation zone (a). Atomic deposition rate as a function of the position of the magnetron into the aggregation zone (b).

Dataset Item 18 (Table). Size distribution data extracted from images 75-76 in Dataset Item 1.

Column 1: Size (nm)

Column 2: Number of Events

Dataset Item 19 (Table). Size distribution data extracted from images 77-78 in Dataset Item 1.

Column 1: Size (nm)

Column 2: Number of Events
Dataset Item 20 (Table). Size distribution data extracted from images $79-81$ in Dataset Item 1.

Column 1: Size (nm)

Column 2: Number of Events

Dataset Item 21 (Table). Size distribution data extracted from images 82-84 in Dataset Item 1.

Column 1: Size (nm)

Column 2: Number of Events 
Dataset Item 22 (Table). Size distribution data extracted from images 85-87 in Dataset Item 1.

Column 1: Size (nm)

Column 2: Number of Events

\section{Concluding Remarks}

We have reported a study on the efficiency of fabricating nanoparticles of magnetic materials (cobalt) using a Multiple Ion Cluster Source. The mean size of the nanoparticles, deposition rate, and atomic deposition rate of the deposits can be adjusted through the tuning of the working parameters such as the power applied to the Co magnetron, argon flux injected to the Co magnetron, total argon flux, and aggregation length of the Co magnetron. The efficiency as a function of applied power follows a nonlinear evolution and it has been shown that nanoparticles with mean size ranging from 4 to $14 \mathrm{~nm}$ can be fabricated. While the increase of total argon flux induces a clear increase of both the mean NPs size and deposition rate, the increase of argon flux injected through the Co magnetron shows a more complex behavior where the biggest NPs are generated at the lowest Ar fluxes. Finally it has been demonstrated that the size and deposition rate increase with increasing aggregation length up to $130 \mathrm{~mm}$ and then decrease. The mean size of the nanoparticles can be fine-tuned by adjusting the aggregation length. In addition, it has been found that those characteristics of the deposits present more complex tendencies than the reported case of a noble metal like silver.

\section{Dataset Availability}

The dataset associated with this Dataset Paper is dedicated to the public domain using the CC0 waiver and is available at http://dx.doi.org/10.1155/2014/584391/dataset.

\section{Conflict of Interests}

The authors declare that there is no conflict of interests regarding the publication of this paper.

\section{Acknowledgments}

This work was supported by the Spanish Ministerio de Ciencia e Innovación and Comisión Interministerial para la Ciencia y la Tecnología (CICYT) under Contract nos. MAT200806765-C02-02, MAT2011-29194-C02-02, and CSD2007-00041 (NANOSELECT). Daniel Llamosa Perez acknowledges the financial support from the Ministerio de Ciencia e Innovación under Contract no. JAEPre-09-01925. The Consejo Superior de Investigaciones Científicas is also acknowledged under Contract no. PIE 201160E085.

\section{References}

[1] M. C. Roco, "International perspective on government nanotechnology funding in 2005," Journal of Nanoparticle Research, vol. 7, no. 6, pp. 707-712, 2005.
[2] R. Ferrando, J. Jellinek, and R. L. Johnston, "Nanoalloys: from theory to applications of alloy clusters and nanoparticles," Chemical Reviews, vol. 108, no. 3, pp. 845-910, 2008.

[3] R. Ghosh Chaudhuri and S. Paria, "Core/shell nanoparticles: classes, properties, synthesis mechanisms, characterization, and applications," Chemical Reviews, vol. 112, no. 4, pp. 2373-2433, 2012.

[4] N. Sanpo, C. C. Berndt, C. Wen, and J. Wang, "Transition metal-substituted cobalt ferrite nanoparticles for biomedical applications," Acta Biomaterialia, vol. 9, no. 3, pp. 5830-5837, 2013.

[5] Y. H. Xu, J. Bai, and J.-P. Wang, "High-magnetic-moment multifunctional nanoparticles for nanomedicine applications," Journal of Magnetism and Magnetic Materials, vol. 311, no. 1, pp. 131134, 2007.

[6] C. Burda, X. Chen, R. Narayanan, and M. El-Sayed, "Chemistry and properties of nanocrystals of different shapes," Chemical Reviews, vol. 105, no. 4, pp. 1025-1102, 2005.

[7] J. Siegel, O. Kvítek, P. Ulbrich, Z. Kolská, P. Slepička, and V. Švorčík, "Progressive approach for metal nanoparticle synthesis," Materials Letters, vol. 89, pp. 47-50, 2012.

[8] G. S. Chaubey, N. Poudyal, Y. Z. Liu, C. B. Rong, and J. P. Liu, "Synthesis of Sm-Co and $\mathrm{Sm}-\mathrm{Co} / \mathrm{Fe}$ nanocrystals by reductive annealing of nanoparticles," Journal of Alloys and Compounds, vol. 509, no. 5, pp. 2132-2136, 2011.

[9] S. Pratontep, S. J. Carroll, C. Xirouchaki, M. Streun, and R. E. Palmer, "Size-selected cluster beam source based on radio frequency magnetron plasma sputtering and gas condensation," Review of Scientific Instruments, vol. 76, no. 4, Article ID 045103, 9 pages, 2005.

[10] S. P. Gubin, Y. A. Koksharov, G. B. Khomutov, and G. Y. Yurkov, "Magnetic nanoparticles: preparation, structure and properties," Russian Chemical Reviews, vol. 74, no. 6, pp. 489520, 2005.

[11] F. E. Kruis, H. Fissan, and A. Peled, "Synthesis of nanoparticles in the gas phase for electronic, optical and magnetic applications-a review," Journal of Aerosol Science, vol. 29, no. 5-6, pp. 511-535, 1998.

[12] K. Wegner, P. Piseri, H. V. Tafreshi, and P. Milani, “Cluster beam deposition: a tool for nanoscale science and technology," Journal of Physics D, vol. 39, no. 22, article R02, pp. R439-R459, 2006.

[13] Y.-H. Xu and J.-P. Wang, "Direct gas-phase synthesis of heterostructured nanoparticles through phase separation and surface segregation," Advanced Materials, vol. 20, no. 5, pp. 994-999, 2008.

[14] J. R. van Ommen, C. U. Yurteri, N. Ellis, and E. M. Kelder, "Scalable gas-phase processes to create nanostructured particles," Particuology, vol. 8, no. 6, pp. 572-577, 2010.

[15] I. M. Goldby, B. von Issendorff, L. Kuipers, and R. E. Palmer, "Gas condensation source for production and deposition of size-selected metal clusters," Review of Scientific Instruments, vol. 68, no. 9, pp. 3327-3334, 1997.

[16] R. C. Flagan and M. M. Lunden, "Particle structure control in nanoparticle synthesis from the vapor phase," Materials Science and Engineering A, vol. 204, no. 1-2, pp. 113-124, 1995.

[17] K. Wegner and S. E. Pratsinis, "Nozzle-quenching process for controlled flame synthesis of titania nanoparticles," AIChE Journal, vol. 49, no. 7, pp. 1667-1675, 2003.

[18] M. Gracia-Pinilla, E. Martínez, G. S. Vidaurri, and E. PérezTijerina, "Deposition of size-selected $\mathrm{Cu}$ nanoparticles by inert gas condensation," Nanoscale Research Letters, vol. 5, no. 1, pp. 180-188, 2010. 
[19] E. Quesnel, E. Pauliac-Vaujour, and V. Muffato, "Modeling metallic nanoparticle synthesis in a magnetron-based nanocluster source by gas condensation of a sputtered vapor," Journal of Applied Physics, vol. 107, no. 5, Article ID 054309, 8 pages, 2010.

[20] C. G. Granqvist and R. A. Buhrman, "Ultrafine metal particles," Journal of Applied Physics, vol. 47, no. 5, pp. 2200-2220, 1976.

[21] K. Sattler, J. Mühlbach, and E. Recknagel, "Generation of metal clusters containing from 2 to 500 atoms," Physical Review Letters, vol. 45, no. 10, pp. 821-824, 1980.

[22] M. T. Swihart, "Vapor-phase synthesis of nanoparticles," Current Opinion in Colloid \& Interface Science, vol. 8, no. 1, pp. 127133, 2003.

[23] S. V. Vladimirov and K. Ostrikov, "Dynamic self-organization phenomena in complex ionized gas systems: new paradigms and technological aspects," Physics Reports, vol. 393, no. 3-6, pp. 175-380, 2004.

[24] J. Kousal, O. Polonskyi, O. Kylián et al., "Characterization of nanoparticle flow produced by gas aggregation source," Vacuum, vol. 96, pp. 32-38, 2013.

[25] L. L. Lazarus, C. T. Riche, N. Malmstadt, and R. L. Brutchey, "Effect of ionic liquid impurities on the synthesis of silver nanoparticles," Langmuir, vol. 28, no. 45, pp. 15987-15993, 2012.

[26] S. Hartner, M. Ali, C. Schulz, M. Winterer, and H. Wiggers, "Electrical properties of aluminum-doped zinc oxide (AZO) nanoparticles synthesized by chemical vapor synthesis," Nanotechnology, vol. 20, no. 44, Article ID 445701, 8 pages, 2009.

[27] T. Mashuri, E. Yahya, and Darminto, "Synthesis of $\mathrm{Fe}_{3} \mathrm{O}_{4}$ nanoparticles from iron sands and effects of $\mathrm{Ni}$ and $\mathrm{Zn}$ substitution on structures and magnetic properties," Journal of Materials Science and Engineering A, vol. 5, no. 9, pp. 182-189, 2011.

[28] H. Haberland, M. Karrais, and M. Mall, "A new type of cluster and cluster ion source," Zeitschrift für Physik D, vol. 20, no. 1, pp. 413-415, 1991.

[29] C. Binns, "Nanoclusters deposited on surfaces," Surface Science Reports, vol. 44, no. 1-2, pp. 1-49, 2001.

[30] I. Shyjumon, M. Gopinadhan, C. A. Helm, B. M. Smirnov, and R. Hippler, "Deposition of titanium/titanium oxide clusters produced by magnetron sputtering," Thin Solid Films, vol. 500, no. 1-2, pp. 41-51, 2006.

[31] V. Bouchat, O. Feron, B. Gallez et al., "Carbon nanoparticles synthesized by sputtering and gas condensation inside a nanocluster source of fixed dimension," Surface and Coatings Technology, vol. 205, supplement 2, pp. S577-S581, 2011.

[32] R. M. Nielsen, S. Murphy, C. Strebel, M. Johansson, I. Chorkendorff, and J. H. Nielsen, "The morphology of mass selected ruthenium nanoparticles from a magnetron-sputter gas-aggregation source," Journal of Nanoparticle Research, vol. 12 , no. 4, pp. 1249-1262, 2010.

[33] G. Baldi, D. Bonacchi, C. Innocenti, G. Lorenzi, and C. Sangregorio, "Cobalt ferrite nanoparticles: the control of the particle size and surface state and their effects on magnetic properties," Journal of Magnetism and Magnetic Materials, vol. 311, no. 1, pp. 10-16, 2007.

[34] W. Lu, D. Sun, and H. Yu, "Synthesis and magnetic properties of size-controlled CoNi alloy nanoparticles," Journal of Alloys and Compounds, vol. 546, pp. 229-233, 2013.

[35] X. D. Zhang, D. Wu, X. Shen et al., "Size-dependent radiosensitization of PEG-coated gold nanoparticles for cancer radiation therapy," Biomaterials, vol. 33, no. 27, pp. 6408-6419, 2012.

[36] J. Y. Park, Y. Zhang, S. H. Joo, Y. Jung, and G. A. Somorjai, “Size effect of RhPt bimetallic nanoparticles in catalytic activity of CO oxidation: role of surface segregation," Catalysis Today, vol. 181, no. 1, pp. 133-137, 2012.

[37] E. L. Roman García, L. Martínez Orellana, M. Díaz Lagos, and Y. Huttel, “Spanish Patent P201030059,” PCT/ES2011/070032, 2010.

[38] L. Martínez, M. Díaz, E. Román, M. Ruano, D. Llamosa P, and Y. Huttel, "Generation of nanoparticles with adjustable size and controlled stoichiometry: recent advances," Langmuir, vol. 28, no. 30, pp. 11241-11249, 2012.

[39] Oxford Applied Research Ltd., http://www.oaresearch.co.uk/.

[40] M. Ruano, L. Martínez, and Y. Huttel, "Investigation of the working parameters of a single magnetron of a multiple ion cluster source: determination of the relative influence of the parameters on the size and density of nanoparticles," Dataset Papers in Science, vol. 2013, Article ID 597023, 8 pages, 2013.

[41] J. A. de Toro, J. P. Andrés, J. A. González, P. Muñiz, and J. M. Riveiro, "The oxidation of metal-capped Co cluster films under ambient conditions," Nanotechnology, vol. 20, no. 8, Article ID 085710, 2009.

[42] J. M. Colino García, "Unidad de pulverización catódica de blancos circulares," Spanish Patent P200900929, 2009.

[43] Nanotec Electrónica S.L., http://www.nanotec.es/.

[44] I. Horcas, R. Fernández, J. M. Gómez-Rodríguez, J. Colchero, J. Gómez-Herrero, and A. M. Baro, "WSXM: a software for scanning probe microscopy and a tool for nanotechnology," Review of Scientific Instruments, vol. 78, no. 1, Article ID 013705, 8 pages, 2007.

[45] K. O'Grady and A. Bradbury, "Particle size analysis in ferrofluids," Journal of Magnetism and Magnetic Materials, vol. 39, no. 1-2, pp. 91-94, 1983.

[46] M. Díaz, L. Martínez, M. M. Ruano et al., "Morphological, structural, and magnetic properties of Co nanoparticles in a silicon oxide matrix," Journal of Nanoparticle Research, vol. 13, no. 10, pp. 5321-5333, 2011. 

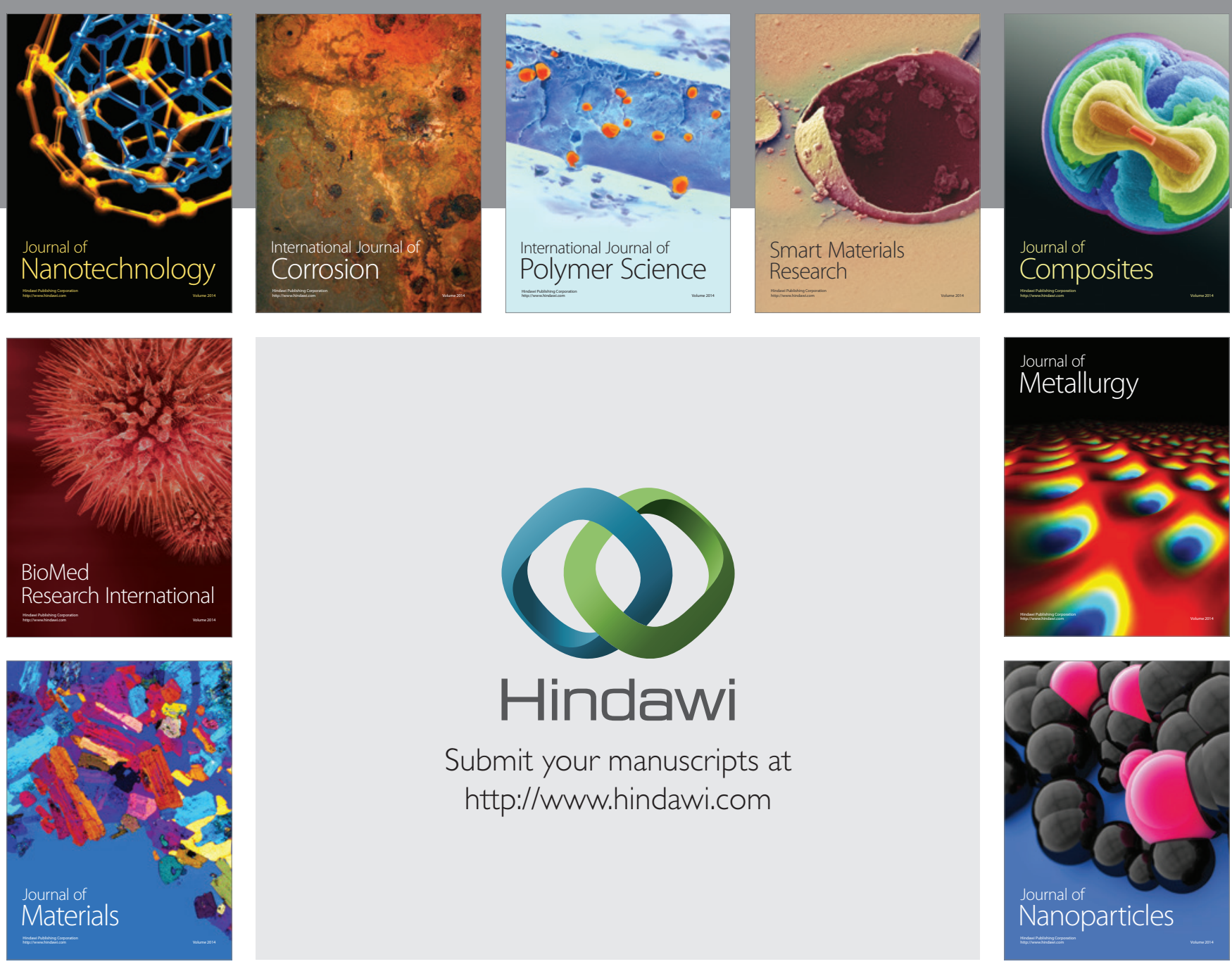

Submit your manuscripts at http://www.hindawi.com
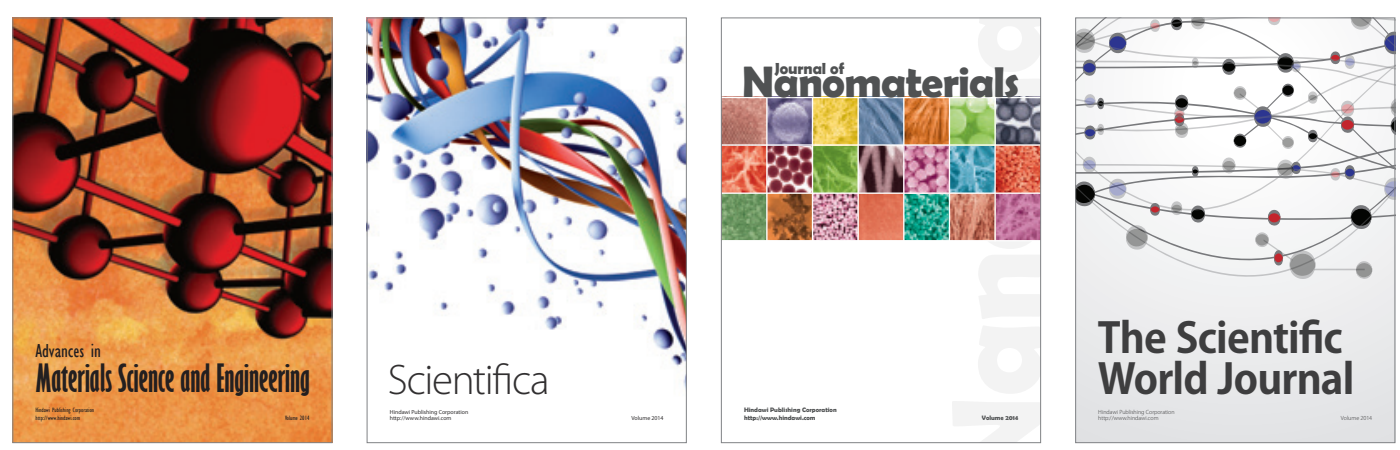

\section{The Scientific World Journal}
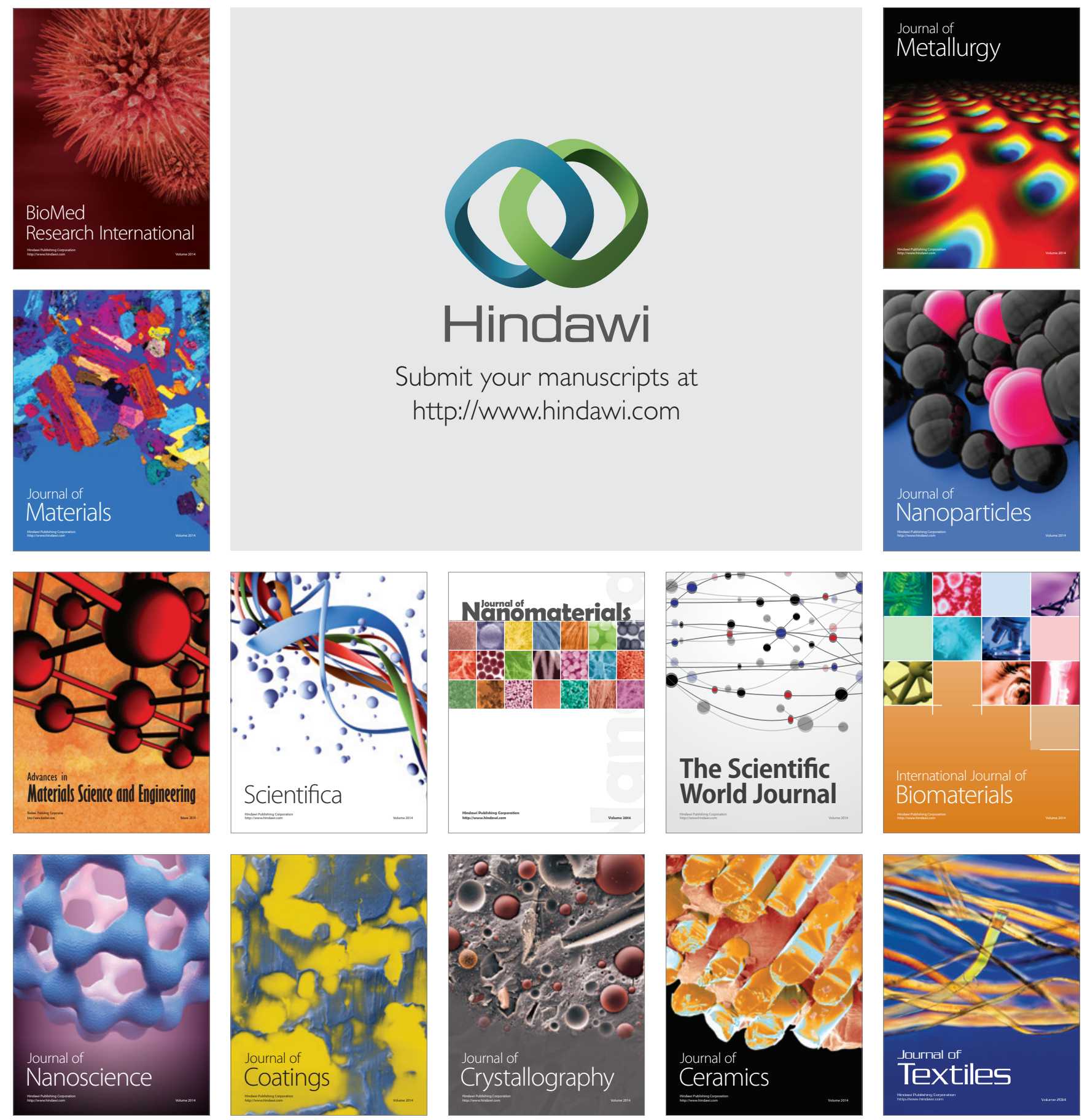